\title{
Entre déjà-dit et jamais-dit. Cancers rares et quêtes d'autorité numérique
}

Between "already and never-said". Rare cancers and digital quest for authority

Juliette Charbonneaux and Karine Berthelot-Guiet

\section{(2) OpenEdition}

Journals

Electronic version

URL: http://journals.openedition.org/aad/5161

DOI: 10.4000/aad.5161

ISSN: 1565-8961

Publisher

Université de Tel-Aviv

\section{Electronic reference}

Juliette Charbonneaux and Karine Berthelot-Guiet, "Entre déjà-dit et jamais-dit. Cancers rares et quêtes d'autorité numérique", Argumentation et Analyse du Discours [Online], 26 | 2021, Online since 14 April 2021, connection on 16 April 2021. URL: http://journals.openedition.org/aad/5161 ; DOI: https:// doi.org/10.4000/aad.5161

This text was automatically generated on 16 April 2021.

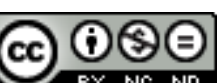

Argumentation \& analyse du discours est mis à disposition selon les termes de la licence Creative Commons Attribution - Pas d'Utilisation Commerciale - Pas de Modification 4.0 International. 


\section{Entre déjà-dit et jamais-dit. Cancers rares et quêtes d'autorité numérique}

Between "already and never-said". Rare cancers and digital quest for authority

Juliette Charbonneaux and Karine Berthelot-Guiet

\section{Introduction}

1 Cet article propose d'interroger la notion de «parent expert » autour de l'analyse de discours numériques portant sur un cancer bien particulier : le glioblastome. Il s'agit d'un cancer rare et peu connu qui, au moment du diagnostic, est souvent très avancé avec un pronostic sombre et des atteintes neurologiques déjà handicapantes. De ce fait, il s'avère que ce sont avant tout des proches qui s'expriment à son sujet au sein des «forums de patients ». Ainsi, cette contribution propose-t-elle de traiter les questions suivantes: comment les discours numériques relatifs au glioblastome font-ils apparaître la construction, par ces parents proches, de figures et positions d'autorité ? Dans quelle mesure leur quête d'autorité en ligne s'articule-t-elle à une prétention à s'affranchir du " déjà-dit » de l'institution médicale, notamment, en revendiquant du «jamais dit »? À partir de l'analyse sémio-discursive des récits et discours tissés dans l'espace numérique d'un corpus de forums francophones dédiés, nous montrerons comment les participants se positionnent par rapport aux acteurs évoqués et en viennent à produire eux-mêmes des formulations d'autorité qui cohabitent avec d'autres formes d'expertise. Le témoignage, encouragé voire demandé par l'organisation communicationnelle des forums sera interrogé comme forme privilégiée d'une autorité qui repose sur une expertise liée au vécu devenant le levier d'autorisation du parent.

2 L'approche que nous proposons est développée au sein d'un site de recherche intégrée sur le cancer ou Siric CURAMUS (Cancer United Research Associating Medicine, University \& Society) lié au site du Groupe Hospitalier Pitié Salpêtrière-Sorbonne 
Université. Les huit Siric labellisés en France par l'Institut du Cancer ont pour mission «d'optimiser et d'accélérer la production de nouvelles connaissances et de favoriser leur diffusion et leur application dans le traitement des cancers " par des programmes intégrés de recherche pluridisciplinaire.

CURAMUS compte trois programmes de recherche intégrée (neuro-oncologie, cancers rares immuno-hématologiques, cancers à instabilité microsatellite) reliés à un programme transversal en sciences humaines et sociales dont les objectifs sont d'aboutir à des évaluations multidisciplinaires, entre expertise technique et préférences du patient, et les questions d'éthique, d'équité, notamment dans l'accès aux soins et de solidarité. Les notions de "démocratie sanitaire" et de "patient expert" sont particulièrement analysées avec les effets d'autorité que de telles dénominations comportent.

4 La notion de "démocratie sanitaire " a été institutionnalisée, en France, depuis 2003 par la loi relative aux «droits des malades et à la qualité du système de santé ». Elle définit la participation des usagers au fonctionnement du système de santé et met en avant les notions d'" agrément des associations et formation des représentants des usagers ». La loi de 2016 de "modernisation de notre système de santé » confirme la démocratie sanitaire en augmentant la place des usagers du système de santé dans les instances régionales et nationales; par ailleurs, elle précise leurs droits (information sur les coûts, les données de santé etc.).

Le « patient expert » apparait dans ce contexte et, tout en posant question, la notion est mise en avant par les institutions qui le définissent comme

le patient qui a acquis de solides connaissances de sa maladie au fil du temps, grâce notamment à l'éducation thérapeutique. Il ne remplace pas le soignant mais il favorise le dialogue entre les équipes médicales et les malades, facilite l'expression des autres patients et contribue à améliorer la compréhension du discours des équipes soignantes.

6 Cette notion de "patient expert » est particulièrement explorée par l'équipe de sciences humaines et sociales du Siric CURAMUS, tant d'un point de vue philosophique, sociologique, anthropologique que communicationnel. En effet, la rareté et le pronostic peu favorable d'une partie des cancers concernés par les trois programmes médicaux conduisent vers des limites complexes. C'est l'une de ces limites que nous nous proposons d'explorer dans cette contribution autour du cas du glioblastome, cancer neurologique rare et peu connu du grand public, qui, au moment du diagnostic, est souvent très avancé avec un pronostic sombre et des atteintes neurologiques déjà handicapantes. De ce fait, le glioblastome est un cancer pour lequel il y a peu d'informations sur le web et, lorsqu'il est abordé dans des "forums de patients " numériques, c'est presque toujours un proche qui prend la parole.

7 De ce fait l'analyse de l'espace public digital du glioblastome, par la grande spécificité et rareté de cette maladie, nous permet d'envisager une sorte d'effet de loupe sur la construction de l'autorité et/ou des autorités en ligne. La façon dont les différentes catégories d'acteurs, institutionnels, médicaux, professionnels, commerciaux, associatifs, les patients et leurs familles apparaissent dans l'espace public numérique du glioblastome permettront une micro-approche de la construction, distribution, négociation des positions et discours d'autorité. Par ailleurs, un travail précis sur les principaux forums de patients, de fait forums de parents ou de proches, permettront d'entrer dans le détail des récits et discours des autorités. Nous verrons comment les 
participants se positionnent par rapport aux acteurs déjà évoqués et comment certains d'entre eux en viennent à produire eux-mêmes des formulations d'autorité qui reposent sur une expertise liée au vécu et relatée par la forme privilégiée du témoignage. En ce point, comme l'énonce Claire Oger, «la «fonction auteur rejoint l'autorité, comme une manière de définir et de régler un champ d'échanges et de discussions, davantage que de contrôler les énoncés produits dans leur sillage » (Oger $2013: 112)$.

de patients en ligne font partie de l'information sur la santé, à destination d'un public que l'on pourrait qualifier de "grand public». Ce type d'information est apparu sur Internet, souvent produit par les internautes, en dehors des instances médicales et étatiques. Elle peut apparaître comme " alternative », introduisant, dans le domaine médical, des prises de paroles proches du militantisme, refusant ou interrogeant les expertises légitimées.

\section{Le corpus}

9 Nous avons construit en plusieurs étapes un corpus de discours en ligne autour du glioblastome dans les "forums de patients ». Dans les deux premières étapes, nous avons entré le terme "glioblastome" dans le moteur de recherche Google, puis le groupe de termes "glioblastome forum ». Ce choix, sans pour autant être exempt de biais, permet d'avoir une idée assez précise des informations, discours et acteurs qui circulent ou interviennent sur le web de langue française à propos de ce cancer rare et peu médiatisé et de comprendre les univers et les acteurs qui lui sont reliés avant d'aller vers les forums dédiés. Dans les deux cas, les quatre premières pages de résultats ont constitué les corpus; sachant que les internautes se contentent en général de consulter la première page de résultat et parfois seulement les premiers résultats, l'amplitude choisie est importante. Dès 2007, la HAS donnait Google comme premier moyen de recherche d'information sur la santé sur Internet ${ }^{1}$. Ce moteur de recherche reste «le serveur le plus communément utilisé que ce soit pour y entrer le nom d'un site particulier, pour y entreprendre une recherche santé par mot-clé ou pour se diriger directement vers Wikipedia $»^{2}$.

De la recherche générale avec le terme "glioblastome", les informations qui apparaissent en premier indiquent essentiellement qu'il s'agit d'un cancer rare, à évolution rapide et fatale pour lequel les soins palliatifs sont tout de suite évoqués. L'univers autour du glioblastome que dessine cette recherche large met l'accent autour d'une maladie peu expliquée et rapidement mortelle. Un traitement par chimiothérapie et un nouveau traitement par vaccin thérapeutique apparaissent également. L'espace public numérique du glioblastome que dessine Google, pour ceux qui n'en connaissent que le nom, présente classiquement d'abord Wikipédia puis des instances médiatiques liées aux questions ou aux professions de santé : Passeport Santé (site d'information en ligne consacré à la santé et aux questions médicales à destination du grand public), Orphanet (portail et fournisseur d'information en accès libre dédié aux maladies et syndromes rares), La Revue Médicale Suisse (publication spécialisée hebdomadaire francophone pour la formation permanente des praticiens) et Le Quotidien du Médecin (journal français consacré à l'information médicale pour les professionnels de santé, médecins et institutionnels); deux associations : la Ligue contre le cancer (organisation non-gouvernementale de lutte contre le cancer reconnue par l'Etat français depuis 
1918) et Imaginformargo (site de l'association Imagine for Margo-children without cancer, association et fondation établies respectivement en 2011 et 2017, dans le but de faire prendre conscience et de lever des fonds pour la recherche contre le cancer chez les enfants), et le laboratoire Roche (groupe pharmaceutique suisse, producteur de l'Avastin, un des composants clé des chimiothérapies contre le glioblastome). Les instances publiques, les hôpitaux, les médecins et les malades sont absents de ces résultats. La Ligue contre le Cancer renvoie vers des forums de patients et rend compte de l'histoire d'une jeune malade.

11 Ce premier paysage numérique du glioblastome esquissé par la recherche faite sur le moteur Google est assez classique au sens où l'information «publique s'intègre dans des logiques socio-économiques ». On trouve bien le secteur public, le secteur associatif institutionnalisé comme plus restreint, des sites marchands (le laboratoire pharmaceutique) et des sites d'information sur la santé que l'on peut qualifier de « tiers nébuleux » (Akrich et Méadel 2010).

Cela se confirme avec les résultats de la requête avec le groupe de termes " glioblastome forum » qui nous a permis de réunir 28 résultats dont huit résultats liés au témoignage ; les sites qui accueillent ces forums de discussion sont Allodocteurs (site lié aux programmes télévisés santé et médecine de France 5), Santé médecine Journal des Femmes (site web autour de la santé lié au Journal des Femmes, production médiatique en ligne créé en 2003), Forum-doctissimo (premier site français, en audience, dédié à la santé et au bien-être), Guérir du cancer (site initié par un oncologue français promouvant un «traitement métabolique »), Forumcancer.ch (site suisse modéré par la Ligue Info Cancer, Ligue contre le cancer suisse), Forum liguecancer.net (site de la ligue contre le cancer déjà présentée), Beauté Test (guide d'achat spécialiste de la beauté créé en 2010) et Carenity (réseau social destiné aux personnes concernées par une maladie chronique créé en 2011). La variété des sites qui abritent ces forums permet d'aller d'univers ouverts à des publics très larges ou vers des cercles plus restreints. Cependant, à l'analyse, la proximité des messages est frappante et rend possible un traitement en commun.

Les échanges recueillis systématiquement réunissent 89 énonciateurs/auteurs de textes singuliers (un post unique) ou récurrents (participation à des conversations en ligne). Ce corpus a ensuite été analysé au moyen d'une approche sémio-communicationnelle par analyse de contenu thématique, analyse du discours, analyse pragmatique, narrative, et sociosémiotique. Elle repose sur la mise en œuvre d'une "plasticité » ou créativité méthodologique car chaque nouvelle réflexion implique la mise en place de combinaisons différentes. La grille d'analyse construite ad hoc repose sur les grandes catégories suivantes: le thème principal ou les thèmes abordés dans la contribution: les aspects factuels (les dates, lieux, traitements etc.), les qualifications de relations (dans la famille, avec l'extérieur, avec les soignants, entre les participants au forum), les qualifications des sentiments, l'embrayage discursif, la modalisation, les emplois de l'impératif et de ses équivalents, les qualifications du parent malade et de son rapport à la maladie, les commentaires "méta » liés au dispositif numérique (commentaire sur l'échange communicationnel et sa qualité, commentaire sur les idées et conseils des autres, état sur des recherches menées en ligne et hors ligne, lien hypertexte vers d'autres sites, remarques sur la pertinence de l'échange, remarques et commentaires proches de l'échange épistolaire pré-numérique, dialogues interpersonnels de l'ordre 
de l'intime, sociabilité numérique commentée), les discours explicites de partage d'expérience, de conseil et/ou d'expertise.

Nous convoquons et mettons en œuvre des méthodes d'analyse du discours pour comprendre des processus de communication complexes autour de la construction d'autorité par les discours en ligne et en saisir la reconfiguration continue. Nous avons tenté d'approcher ces fabriques numériques de l'autorité autour du glioblastome comme des figures d'action appréhendables dans les interactions des aidants, parents et patients sur les forums numériques. Nous cherchons comment certains discours se légitiment et se trouvent légitimés au sein des forums. En effet, au cours de ces échanges, certains scripteurs vont être institués par les autres, par eux-mêmes et, enfin, par le dispositif numérique et ses spécificités; l'autorité venant, qu'il y ait prétention ou pas. La crédibilité des locuteurs se construit dans les forums sans position institutionnelle par le jeu des dimensions discursives et contextuelles. L'expérience devient ou fournit la compétence; un savoir-faire d'aidant ou d'accompagnant devient la source d'une autorité.

\section{Rapport au(x) savoir(s) : les insuffisances du « déjà- dit »}

15 Les architextes ${ }^{3}$ des forums constituent une première manifestation autoritaire en ce qu'ils prescrivent aux locuteurs des possibilités contraintes d'expression numérique (Jeanne-Perrier 2006). Ainsi, les textes des parents ou patients prennent la forme de fragments de différentes tailles qui, mis en série, forment un « diatexte $»^{4}$ (Fournout 2009) à l'écran. Ce diatexte se caractérise par sa double dimension intertextuelle : la première arrime chacun des textes individuels à ceux des autres participants; la seconde, partagée, ouvre pour sa part sur l'évocation de discours tenus, entendus et recueillis « en-dehors » du forum. Ce second type d'intertextualité, que nous qualifions de "déjà-dit », constitue le cœur de l'analyse menée dans ce premier mouvement, visant à saisir le rapport entretenu par les locuteurs au(x) savoir(s) sur le glioblastome portés par différentes incarnations potentielles de l'autorité médicale et à comprendre comment ce rapport vient légitimer cette autorité mais aussi mettre en lumière certaines de ses fragilités.

\subsection{Rapport aux figures médicales}

16 «Les patients qui s'expriment sur Internet sont très soucieux de respecter le partage des rôles et de ne pas empiéter sur les prérogatives du médecin ", notaient Akrich et Méadel (2010: 45). Il en va de même dans le cas ici étudié : ni les malades ni leurs parents ne viennent remettre en cause l'autorité présumée du savoir médical et de celui qui l'incarne. Au contraire, la manière dont est évoquée de façon transversale la figure du médecin participe pleinement de la construction de son autorité. Cette figure apparaît ainsi comme référence dans les échanges en ligne. Cette référence peut se faire précise, lorsque le médecin est nommément identifié comme une ressource, voire une source d'espoir ${ }^{5}$ : «J'ai réussi à avoir un RDV avec un spécialiste des glio- : M. XX à une clinique de Marseille ", écrit par exemple Laura stehlin ${ }^{6}$. C'est également le cas lorsqu'est formulée l'expression d'une reconnaissance, telle celle affichée par Sky51li : 
«Je dois la vie a l'homme qui ma opéré, le Proffesseur Y au CHU de Limoges, c'est l'un des meilleurs $»^{7}$.

Plus encore, la figure du médecin fait " référence » lorsqu'elle est évoquée en tant que catégorie générique. «Il existe bien un grand chirurgien qui peut faire quelque chose », conseille Henri à Yany au sujet de la maladie de son mari tandis que Ultra-Marine répond à cette même locutrice que «ce type de tumeur nécessite d'être pris en charge par des neurochirurgiens très spécialisés $»^{8}$. Plus qu'une personne donnée, la référence cible et légitime alors, à travers la figure soignante, l'institution médicale dans son ensemble. Témoignent également de cette confiance des discours évoquant pour leur part le champ de la recherche médicale tout entier : « Espérons que la science avance et qu'il puisse guérir celle-ci!» s'exclame ainsi olf $393^{9}$. Forte de cette imbrication d'échelles - discipline, institution, personne - dans lesquelles elle est perçue et mentionnée comme référence, la figure médicale se voit bien renforcée comme autorité « de fait $~^{10}$ dans les forums analysés (Lamy $2017: 43$ ).

Cette confiance, forme de reconnaissance et condition de possibilité de l'autorité, n'empêche toutefois pas les scripteurs d'exprimer une forme d'amertume liée à ce qu'ils perçoivent comme autant d'impossibilités discursives du corps médical. « Ne t'attends pas a de l'optimisme du côté hospitalier, prévient TexasNétoile, ils ne peuvent se permettre de donner ne serais-ce qu'un soupçon d'espoir dans cette maladie, [...] et même mon neurochir, il reste toujours trés neutre et prévenant malgré mes résultats satisfaisants $»^{11}$.

\subsection{Expression d'un manque d'informations}

Antoine Spire et Rollon Poinsot évoquent la potentielle "conspiration du silence", ressentie par les patients et qui « enveloppe l'annonce jusqu'à l'étouffer, générant une souffrance pour tous » $(2007: 163)$. Dans le cas des forums étudiés, il y a bien souffrance exprimée vis-à-vis d'un "silence " informationnel; en revanche, il n'est pas perçu comme une conspiration, terme qui implique une intentionnalité, mais plutôt comme une impuissance partagée, face à l'opacité qui entoure cette maladie rare. « Mon papa a un glioblastome aussi depuis 2012. [...] Si son traitement s'arrête malheureusement on ne peux pas savoir combien de temps il lui reste. ça peux aller de quelques jours, à quelques semaines, à quelques mois ", écrit ainsi Maud avant de clôturer son récit par la reprise anaphorique du même regret: "On ne peux pas savoir $»^{12}$. Le fait que les parents de patients soient au fait de cette impuissance, partagée par les médecins, peut expliquer l'absence de reproches à leur égard et l'aspect unanimement compréhensif des propos exprimés à leur sujet.

Cette impossibilité de savoir, liée à la rareté de la maladie, se voit également attribuée par les scripteurs à la pauvreté des discours institutionnels et médiatiques susceptibles de l'expliciter. Laure raconte ainsi la quête d'informations entreprise suite à l'annonce de la maladie de son père : «Avec ma famille nous avons écumé le service d'oncologie à la recherche de groupes de parole sur ce cancer, en vain. Oui, rien n'existe en province pour les patients touchés, on ne parle pas de ce cancer, parce qu'il n'est pas vendeur, parce que le pronostic est sombre $»^{13}$. «Les sources d'information sur internet sur cette maladie sont peu nombreuses et particulièrement angoissantes sur les possibilités de rémission d'autant plus que les cas de récidive paraissent nombreux ", regrette pour sa part Espoir 04 $4^{14}$. 
21 Ce non-savoir qui participe à la souffrance ressentie s'exprime également par un recours généralisé à la forme interrogative. Patients et parents partagent ainsi, à " plume haute ", leurs questionnements vis-à-vis de la maladie, de son diagnostic et de ses évolutions, à l'instar de $\mathrm{Kika}^{15}$ : «Mardi, nous avons appris que mon papa avait un Glioblastome frontal de $6 \mathrm{~cm}$, le médecin généraliste a utilisé le terme de : rare. Je me rends compte en lisant certains articles sur internet que ce n'est pas si rare que ça... elle est frontale, est-ce que c'est ça la rareté?»

\subsection{L'appel aux témoignages pour combler les manques du « déjà- dit » médical}

Les questionnements ainsi formulés trouvent leur prolongement dans une dimension complémentaire communément partagée sur les forums : l'appel à combler le manque d'informations par des récits testimoniaux qui vont instituer l'expérience personnelle en une forme de savoir. À la quête, parfois désespérée, d'informations vient ainsi s'ajouter une requête adressée aux autres participants qui construit, déjà, a priori, leur légitimité à pouvoir dire. L'énoncé de cette requête revêt deux formes qui peuvent cohabiter ou qui, en tout cas, ne sont pas exclusives l'une de l'autre. La première a trait à la traversée de la maladie elle-même. «J'appréhende surtout la suite car j'ai bien compris qu'on en guerri que très rarement. J'aimerais avoir vos témoignages pour ceux qui passent ou sont passés par là...", lance Marineke ${ }^{16}$. Les questions peuvent également se faire plus précises et viser directement les modalités de traitement expérimentées par les participants ou leurs parents. "J'aimerais savoir si qq a été traité par le DEPATUX pour glioblastome stade 4 récidivant », demande ainsi Cris ${ }^{17}$.

Enfin, et c'est là la seconde forme de requête principale, les locuteurs interrogent et s'interrogent sur la conduite de la vie quotidienne dans la maladie et sur le registre très personnel des relations à entretenir avec ses proches au long de cette expérience. "Que dois je faire envers mon copain? je ne peux me permettre d'être égoiste et le laisser rester à mes côtés, [...] c'est quoi la décision la plus sage à prendre vous qui ont déjà passé par là; qui ont survécu à cette maudite maladie! », implore Imp26jc ${ }^{18}$.

Alice Krieg-Planque caractérise les discours d'autorité comme « des discours dont la linéarité a été travaillée de manière que ni l'imprévu ni les débordements n'y trouvent plus place » (2015: 115). La figure d'autorité médicale n'est ici nullement remise en question. En revanche, son discours se voit, lui, bousculé par les limites et manques énoncés par les participants aux forums. Le "déjà-dit» - qu'il soit médical, institutionnel ou médiatique - s'avère ainsi insuffisant face à l'ampleur du bouleversement vécu. C'est précisément cette insuffisance qui va entraîner les locuteurs à rechercher des débordements discursifs, qui s'éloigneront des formats classiques de l'autorité médicale pour en produire d'autres qui relèvent de l'ordre du « jamais-dit » et permettant d'accéder à l'échange symbolique évoqué par Baudrillard. Dans L'Échange symbolique et la mort, celui-ci expose en effet

l'exigence aberrante et irrecevable du malade (et du mourant) de fonder un échange sur cette différence - non pas du tout de se faire soigner et rectifier, mais de donner sa maladie, et qu'elle soit reçue, donc symboliquement reconnue et échangée, au lieu d'être neutralisée dans la mort technique hospitalière et dans cette survie strictement fonctionnelle qui s'appelle la guérison et la santé ( 1976 : 299). 


\section{Rapport à l'expérience, conquête et normalisation d'un « jamais-dit »} glioblastome et surtout les accompagnants, se trouvent donc face à un déjà-dit pauvre et technique, difficile à trouver et à évaluer bien qu'il bénéficie sans conteste de l'autorité médicale. La recherche qui mène alors les parents jusqu'aux forums de patients est une quête du semblable, du comparable, soit de la narration de l'expérience des autres pour recueillir dans les témoignages des éléments « inédits " puisque jamais dits par les instances déjà connues (Jolivet 2010). L'expérience, dont rend compte essentiellement le témoignage, ouvre la porte à la constitution d'un « dit en commun » qui permet de construire de l'entre-dit, de le constituer en discours circulant par la récurrence. La convergence, la résonance créent une reproductibilité du discours d'expérience et contribue à le normaliser pour l'amener à un degré d'autorisation suffisant.

C'est en même temps une autorité et un lien qui émerge du partage permis par les dispositifs numériques dits «forums ». La création de lien par des dispositifs à distance se retrouve dans les dispositifs dits d'éducation thérapeutique qui reposent notamment sur des appels réguliers entre soignants et patients. Pouvoir dire et échanger sur l'expérience de la maladie fait partie de leurs bienfaits majeurs (Mayère 2018). En effet, au-delà de l'attention, c'est un lien qui s'établit et est documenté, à travers ce que l'éducation thérapeutique, décrit comme une « continuité construite par les infirmières grâce à leurs consignations dans les dossiers patients » et, dans les forums de patients, par l'archive ouverte que constitue l'historique des échanges (Marcoccia 2004). La conscience qu'ont les participants de cet archivage fait partie de la première mise en autorité de ces discours. Ce qu'ils écrivent, leur expérience, restera et pourra être consultée par d'autres qui se trouvent ou se trouveront face aux mêmes questions, des mois ou des années plus tard. Lorsque la pathologie est rare et que les informations sont difficiles à atteindre et à collecter, comme c'est le cas pour le glioblastome, on assiste à une mise au travail des parents et parfois patients dont les effets sont très différents de ceux de l'éducation thérapeutique; les scripteurs des forums, tout en se disciplinant, se donnent les moyens de se sentir davantage en action (Mayère 2018).

L'échange et la mise en commun des expériences permettent aux parents de construire une "expertise profane" qui passe d'une expertise dite faible, "c'est-à-dire l'appropriation d'un savoir expert », à une expertise profane au sens fort - soit "un savoir du profane en tant que profane» (Broca et Koster 2011: 112). Les scripteurs concourent à la rédaction d'un jamais-dit rendu nécessaire autant que légitime par la technicité du discours de soignants (voir supra) . Le motif de l'annonce du diagnostic présent dans la plupart des primo-interventions dans un forum en ligne est, en même temps, dans la plupart des cas, ce qui justifie la prise de parole en ligne. L'annonce transforme les rôles et places discursifs, à partir de cet événement certains deviennent patients et d'autres parents (Berthelot-Guiet et Charbonneaux 2020).

À partir de là, une autre autorité peut se construire, non concurrente de celle des médecins, nécessaire au fonctionnement quotidien brutalement transformé. Cette autorité vient des usages quand celle des médecins vient de la source (Romeyer 2008). La forme même du témoignage, telle qu'elle est encouragée voire prescrite par/sur les forums, devient levier d'autorisation du parent, celle de manifester une autre autorité

Argumentation et Analyse du Discours, 26 | 2021 
qui prend sa source dans le vécu de la maladie. Les témoignages construisent un savoir partagé par les familles des patients qui porte sur la maladie, les traitements, le vécu, les émotions... Une répartition des savoirs se déploie, entre «les savants ", à savoir les soignants et une nouvelle catégorie, en cours de production, de "sachants", à savoir " ceux dont la connaissance a été acquise dans l'action, par le savoir vécu transmis, mais aussi conquise en réponse à une question, à un problème personnel particulier " (Méadel 2010 : 111).

Ces savoirs sont certes profanes mais, par le dispositif du forum numérique, par l'échange des expériences et leur agrégation, produisent une expertise et représentent une autorité. Grâce à ces forums, les parents et patients passent du statut de « ceux qui ne connaissent pas » au statut de ceux « qui ont des connaissances et des points de vue qui peuvent être aussi établis, aussi fondés, aussi rationnels, aussi utiles que ceux des scientifiques" (Epstein, Méadel 2010: 112). Et ce, d'autant que le forum assure la diffusion, l'archivage, la constitution d'une base de données.

La notion d'expertise profane comme expertise expérientielle (Akrich et Rabeharisoa 2012) se dessine dans notre corpus, comme en témoigne le message de Laureteste :

Il y a moins d'un an je ne connaissais pas cette maladie, maintenant qu'elle a pris mon Papa, j'aimerais la faire connaitre du grand public et votre émission pourrait permettre cela. Toutefois, je pense qu'il ne faudrait pas l'aborder uniquement du point de vue «médical » mais aussi montrer les dommages collatéraux d'une telle maladie (répercussions sur la famille, le quotidien diminué ...) ${ }^{19}$.

(1)

l'estime de soi (Mayère 2018). En effet, le combat parallèle à celui des soignants mené par les parents s'articule, pour ces derniers, à un fort investissement du champ du soin et de l'accompagnement quotidiens et des émotions qu'ils engagent. L'hyper-quotidien énoncé par les parents se compose aussi d' «affects numériques", entendus comme « une capacité à ressentir ou faire vivre des émotions » (George et Julliard 2018). Dans le cas du glioblastome, les parents deviennent sujets-acteurs de la maladie à travers le fait même de signifier des émotions à son propos. L'expression des émotions provient

Argumentation et Analyse du Discours, 26 | 2021 
de leur autorisation par le système du forum. Le témoignage comme discours d'autorité de l'expérience entre dans le système général du web qui construit la crédibilité des participants et produit une « autorité des méritants» (Candel et Gkouskou-Giannakou 2017) comme l'énonce le message de Lou à Thyli : « bravo pour l'effort que tu fais pour ton papa bon courage et bcp de patience $»^{22}$.

Dans leurs échanges, les parents produisent des biographies individuelles proches de l'exemplum, dans la mesure où, par des micro-narrations, des "détours narratifs » obligés, elles permettent d'embrayer le discours et de produire un récit singularisé et singularisant qui s'articule à une valeur collective par la création d'une sorte de répertoire commun aux participants (Abiven 2016). L'exemplum, la biographie individuelle entre dans le récit collectif d'abord parce que l'individuel, mis en série dans une micro-forme, devient collectif et permet d'aller vers l'exemplarité pour d'autres et pour la société. Les biographies rendent possible un "acte Monument " dressé par le parent scripteur à son parent malade, "l'acte qui fait reconnaître " (Barthes 2009). Ensuite parce que ce traitement micro-narratif s'étend du patient à sa famille qui est, elle aussi, idéalisée, la figure du combattant lui est étendue comme dans la réponse de And $31 \mathrm{ku}$ à Luna67110 23 : «Il sent qu'on a encore besoin de lui car toute ma famille, ma mère, moi, on le soutient énormément. Je pense qu'il ne faut jamais abandonner ». Les biographies érigent des familles racontées, unies autour du parent malade, sans heurts, sans conflits et sans référence aux relations et aux conflits antérieurs à la maladie. Elles font preuve de courage, entourent, donnent force. Elles produisent une valeur exemplaire et érigent un Monument familial qui peut entrer dans le cercle des autres histoires pour créer les histoires interfamiliales (BerthelotGuiet et Charbonneaux 2020).

Ces monuments familiaux font autorité par la stabilisation même de leur forme et la normalisation énonciative qu'ils présentent. Partis d'énoncés individuels sous le joug de l'énonciation du forum numérique, les messages des parents de malade du glioblastome se combinent et se formulent de manière à entrer dans le champ du conseil, voire de l'injonction, de formes autoritaires travaillées "de manière que ni l'imprévu ni les débordements n'y trouvent plus place » car «les discours d'autorité sont constitués, à différents égards, d'énoncés stabilisés » (Krieg-Planque 2015).

Les discours d'autorité que nous explorons se produisent donc largement par l'autorisation que permet le dispositif numérique lui-même car tout discours d'autorité s'inscrit dans les «matérialités discursives" (Candel et Gkouskou-Giannakou 2017, Monte et Oger 2015). Loin d'être profanes du dispositif, certains des scripteurs montrent qu'ils sont fortement conscients de ses spécificités, capables de questionner ce que les forums permettent ou rendent difficiles dans la petite fabrique de l'autorité.

\section{En lisant, en écrivant : procédés d'autorisation de l'expression numérique}

En plus des motifs précédemment évoqués, les récits et témoignages donnent à lire et à appréhender le rôle joué par la forme même du forum et par l'expression numérique dans les parcours de soin et de vie. Il n'est ainsi pas rare que le forum soit évoqué comme faisant pleinement partie des histoires racontées. "Je me souviens de mon arrivée sur ce forum", raconte par exemple Lip $26^{24}$. "Je remonte mon poste... un besoin de m'éloigner de ce genre de forum... cela fait un an et demi que je me bats 
contre ce foutu cancer ", écrit quant à elle ElodiePM pour expliciter les raisons d'une " absence " en ligne ${ }^{25}$. Etienne Candel et Pergia Gkouskou-Giannakou considèrent que "l'emprise des dispositifs, d'une part, et la prise des utilisateurs sur leurs fonctionnalités et leurs opportunités, d'autre part, composent l'autorité, ou plutôt les situations d'autorité, comme des phénomènes dynamiques à la rencontre de médiations hétérogènes " (2017). Dans cette perspective, il s'agit, dans ce dernier mouvement, d'analyser ce que disent les utilisateurs du ou des dispositifs numériques et de comprendre ainsi en quoi le "processus d'autorisation » de la figure du parent passe également par un processus de légitimation des formes qui lui permettent l'expression de l'expérience et par la construction d'un ethos d'expert en terrain numérique.

\subsection{Réflexions sur l'écriture : figures de parents-auteurs}

Les parents ou patients légitiment leurs prises de parole au long d'un processus d'expertise qui n'est pas strictement limité à l'expérience de la maladie mais qui s'étend aussi, quoique plus incidemment, aux modalités d'expression et de communication sur les forums. Ce processus d'autorisation se constitue dans un métadiscours sur leur propre pratique d'écriture, ce qui a pour effet d'affirmer leur ethos discursif d'auteur. Ainsi de Claelia qui avance « je me permets de vous conseiller le groupe facebook Glio et nous, j'y écrit souvent et cela me fait beaucoup de bien ${ }^{26}$.

Ce processus d'autorisation de la figure du "parent-expert " se construit par ailleurs à travers l'extension du registre du conseil, parfois autoritaire, et, cette fois, étendu au «comportement numérique» que pourrait ou devrait endosser l'un ou l'autre des locuteurs. «J'ai pu voir que sur le forum il y a plusieurs personnes atteintes de la même pathologie que toi, soit un glioblastome. Ils ont ouvert leur propre file, joins toi à eux ou crée la tienne ", recommande de la même façon Lina 303 à Elodiepm ${ }^{27}$. L'autorité se dessine enfin à travers des interventions actives sur les modalités d'expression des autres membres. Doko 2 s'adresse ainsi à Imp26Jc: «J'éspère que d'autres personnes vont te soutenir et répondre à tes posts sois forte je fais remonter ta file $»^{28}$.

\subsection{Prescriptions de lecture : formes du dialogisme numérique}

41 Sur Internet, pratiques d'écriture et de lecture sont étroitement mêlées, voire indissociables si ce n'est, comme ici, pour les nécessités de l'analyse (Souchier et al. 2019). Les parents ou patients qui interviennent sur les forums sont donc toujours à la fois auteur et lecteur. Observer cette seconde dimension identitaire permet également de préciser le processus d'autorisation étudié, dans la mesure où la lecture affichée mais aussi prescrite joue un rôle non négligeable dans la légitimation de l'expertise.

L'auteur-scripteur en terrain numérique est ainsi aussi un lecteur du numérique et le fait savoir: "D'après ce que j'ai pu lire sur internet, l'espèrence de vie ppur cette maladie n'est pas longue ", écrit Marinekle au sujet de son père ${ }^{29}$. La mise en avant de l'activité de lecture se fait plus active encore lorsque vient s'y ajouter une dimension évaluative, les participants n'hésitant pas à exprimer leurs jugements vis-à-vis de tel ou tel espace d'expression numérique. «Attention aux forums grand public qui sont très démoralisants ", prévient par exemple Ultra-marine ${ }^{30}$. dialogique, sémiotisée dans les échanges par la fréquence d'apparition de cette « petite 
forme $»^{31}$ qu'est le lien hypertexte ou « signe passeur $»^{32}$. Les prises de parole analysées peuvent ainsi être envisagées comme autant de "discours hypertextualisés", soit comme « discours augmenté par la présence d'un lien hypertexte renvoyant vers un autre discours » (Simon 2016:7). À la posture affichée de lecteur du numérique/sur le numérique vient ainsi s'arrimer une dimension plus active encore, consistant à encourager cette pratique chez les autres participants par des « tentatives d'imposition de lectures préférentielles » (Saemmer 2016).

Dans cette perspective, on peut considérer l'inscription de «signes passeurs » dans les textes des parents et patients comme participant pleinement de leur processus d'autorisation. D'autant plus que les locuteurs ne se contentent pas de mentionner les liens, ils accompagnent ce geste scriptural d'un discours d'accompagnement dans lequel on retrouve le registre du conseil. Celui-ci peut aller de la simple proposition "Si vous souhaitez échanger avec d'autres personnes concernées par cette maladie, vous pouvez vous rendre sur le groupe privé « Le glio et nous » sur Facebook.. » écrit par exemple Emilie $^{33}$ - à une forme de prescription au ton nettement plus affirmée, à l'instar de la quasi-injonction de Marion : " allez plutôt sur ce groupe là, on parle de tout très librement https://www.facebook.com/groups/2198280497072170/ $\underline{\Perp}^{34}$.

Ces « lectures préférentielles », pour reprendre le terme d'Alexandra Saemmer (2016), englobent de manière privilégiée, on le voit aux exemples précités, des écrits avant tout composés par et pour le numérique. Les liens proposés/imposés dans les forums font d'une part signe médiateur vers d'autres textes du même espace, dans une forme de dialogisme « interne » qui compose au forum sa légitimité en montrant son historicité (la longévité devient alors gage de fiabilité). C'est le cas du propos émis par Frédéric, lorsqu'il écrit: "Aujourd'hui je mets à jour mon témoignage de fin novembre 2016 concernant le Glioblastome que l'on m'a découvert en Mars 2015 (voir le témoignage pour l'histoire...). »

Les « signes passeurs » dirigent également vers d'autres dispositifs socio-numériques, à commencer par le réseau Facebook, peut-être parce qu'il présente une proximité rassurante dans les formes de lecture et d'écriture prescrites (la "page »/la «file»). Bet48iz indique par exemple dans sa réponse à Imp26jc : « Mon beau fils 18 ans est dans le même cas. Il suit un traitement d'immunotherapie en Allemagne. Vous pouvez suivre son combat sur la page facebook: le sourire d'Alain Junior. Tu auras également des renseignements sur ce traitement. N'hésite surtout pas à aller sur la page $»^{35}$.

Les liens renvoient, enfin, vers un «extérieur» plus élargi encore, lorsque des scripteurs ouvrent leurs textes vers des références issues du milieu médical. «Je vous invite à vous renseigner sur les travaux du Docteur Z. sur les causes métaboliques du cancer. Vous pouvez en trouver certains en libre accès, il a ouvert les droits pour qu'un maximum de personne puisse les lire ", expose ainsi Cracov02 avant d'ajouter le lien correspondant assorti de celui d'une association liée au thème évoquée ${ }^{36}$.

On voit à ce dernier exemple comment la prescription de lecture engagée par le discours hypertextualisé participe, à son tour, du maintien de l'autorité médicale dans sa position de référence. Le discours hypertextualisé constitue en effet une pratique de la citation, laquelle « fonctionne comme un rappel du statut des locuteurs investis de l'autorité d'un pouvoir-dire» (Mouillaud et Tétu 1989: 138). Ceci peut d'ailleurs expliquer la participation très ponctuelle et apparemment bienvenue de locuteurs qui n'hésitent pas à se présenter comme médecin et à exposer à ce titre leur savoir, dans une forme elle-même hypertextualisée. « Nous vous soutenons dans vos épreuves. Des 
articles ont été réalisé sur la recherche et l'avancement des thérapies concernant les tumeurs cérébrales", écrit ainsi Camille identifié comme "médecin généraliste Nord $»^{37}$.

Dans son analyse des savoirs profanes en ligne, Cécile Méadel écrivait ceci :

Comme toute connaissance, le savoir profane ne se réalise pas exclusivement dans des pratiques implicites ou informelles; il passe par des dispositifs, des façons de faire sens, des supports, des bases de données, des textes... et aussi, a minima, par la restitution de recherche d'informations, l'exploitation des données existantes, le recueil de documents, la diffusion de renseignements, de messages, de résultats $(2010: 111)$.

On a vu, au long de ce dernier temps, comment l'ethos discursif des locuteurs s'affirmait à travers la "fabrique des connaissances", laquelle "commence dès lors que la recherche et la collecte d'informations font l'objet de reprise et de diffusion » (ibid.). Plus encore, dans la double perspective représentationnelle proposée par Louis Marin (1994), les pratiques d'écriture et de lecture, telles qu'elles s'affichent et s'affirment à l'écran, produisent un effet d'objet - la production de ces « savoirs profanes » - et, dans le même temps à travers elle, un effet de sujet, à savoir l'ethos d'un participant capable d'identifier, sélectionner et prescrire et ces savoirs et les modalités de communication susceptibles de les valoriser encore davantage.

\section{Conclusion}

51 L'étude menée ici visait à saisir comment, dans le cas du glioblastome, la médiation de l'écriture numérique pouvait favoriser la position d'autorité et la reconnaissance du parent comme expert potentiel de et dans cette maladie caractérisée par sa rareté. L'analyse de ce " processus d'autorisation » a révélé comment les forums permettent au parent et, dans de plus rares cas, au patient, d'échapper, le temps de l'écriture/lecture à " cette mort anticipée à laquelle on l'a condamné ", à " cette neutralité dans laquelle on l'enferme en échange de guérison " (Baudrillard 1976 : 299).

Cette position et la possibilité de l'échappée qui la permet est bien sûr tributaire des conditions d'expression prescrites par ces "outils d'écriture aux pouvoirs exorbitants » (Jeanne-Perrier 2006) que sont les architextes des forums. C'est à l'intérieur de ces cadres contraignants qui imposent leur « ordre du discours » que vient se déployer une information véritablement " compensatoire » dans la mesure où elle vient combler ce qui est ressenti comme un vide. Cette information est une construction bricolée par les familles pour les familles dans l'interdiscours des formes du dialogisme numérique propre aux forums, une double échappée qui souligne les insuffisances des paroles légitimes et interroge fondamentalement la question de l'autorité quand elle-même semble en défaut de savoir.

53 Les interactions en ligne des patients et parents autour du glioblastome ne constituent pas pour autant la remise en question d'un monopole sur le savoir ou une « remise en cause du pouvoir médical "; elles sont la manifestation discursive de la recherche d'un accès au savoir et à l'expression pour une pathologie qui excède le déjà-dit ou le dicible des médecins. Cette recherche ou quête d'un "jamais-dit» fait émerger un genre d'expression privilégié : le témoignage. C'est au sein de cette forme que vient se tisser une "expertise profane", soit "un savoir, distinct de celui possédé par l'institution médicale, qui trouverait son origine dans l'expérience existentielle, physique, de la 
maladie, et se construirait au travers de formes d'apprentissage largement empiriques » (Broca et Koster 2011). Le fait de vivre ou d'avoir vécu cette expérience construit donc une autorité testimoniale, à l'échelle de l'individu qui peut ainsi reprendre la main sur son propre récit biographique et celui de sa famille mais aussi à l'échelle d'une communauté glioblastome réunie dans le partage d'émotions.

«L'existence d'un sentiment communautaire, au sens de buts et de valeurs partagés, ne prémunit pas contre l'émergence de formes d'inégalités quant au statut des participants", notaient Broca et Koster avant d'ajouter que «le fait de devoir s'exprimer par écrit demeure ainsi pour de nombreuses personnes un frein majeur à la contribution aux échanges " (2011). Les discours étudiés dans cet article ne permettent pas d'identifier des inégalités qui émaneraient des positions et dispositions sociales des participants, ceux-ci n'évoquant presque jamais leurs conditions matérielles d'existence. En revanche, le dialogisme des témoignages élève certains échanges et, ce faisant, fait aussi émerger des locuteurs plus « autoritaires » et autorisés que d'autres et, à travers eux, une scénographie assez normée du ton et des messages.

Finalement, l'expression demeure tributaire d'une «technique » d'écriture en terrain numérique qui, en s'affichant et en s'affirmant, maintient le savoir profane des parents éloigné du pouvoir, si l'on considère avec Didier Fassin que «le savoir apparaît ainsi d'autant plus lié au pouvoir qu'il est abstrait et secret. Il en est d'autant plus séparé qu'il est technique et profane" (1996: 141). Cela étant, les discours ne laissent percevoir aucune manifestation, aucun désir, d'extension de la sphère d'action explorée sur les forums vers les parcours de soin. Le fait de pouvoir devenir témoinauteur semble leur suffire à faire émerger la «survie " symbolique que Baudrillard qualifiait d'« opération fondamentale de la naissance du pouvoir» (1976:212). Cette dimension demanderait toutefois à être éprouvée, par exemple, par l'ouverture de l'analyse à d'autres terrains d'expression numérique, tels les réseaux sociaux type Twitter ou Instagram.

\section{BIBLIOGRAPHY}

Abiven, Karine. 2016. L'exemplum : un modèle opératoire dans la lettre familière ?", Exercices de rhétorique [En ligne] 6 | 2016 https://journals.openedition.org/rhetorique/431 (consulté le 08/10/2020)

Akrich, Madeleine \& Vololona Rabeharisoa. 2012. «L'expertise profane dans les associations de patients, un outil de démocratie sanitaire », Santé Publique 24.1, 69-74

Barthes, Roland. 2009. Le Journal de deuil (Paris : Seuil)

Baudrillard, Jean. 1976. L'Échange symbolique et la mort (Paris : Gallimard)

Berthelot-Guiet, Karine \& Juliette Charbonneaux. 2020. « Vers une entraide numérique intergénérationnelle? Le cas du Glioblastome sur les forums de discussion en ligne ». (Revue française des sciences de l'information et de la communication [En ligne] 19 | 2020, http:// journals.openedition.org/rfsic/8642 (consulté le 10/10/2020) 
Broca, Sébastien \& Raphaël Koster. 2011. « Les réseaux sociaux de santé. Communauté et coconstruction de savoirs profanes ", Les Cahiers du numérique 7.2, 103-116

Candel, Étienne, Valérie Jeanne-Perrier, Emmanuël Souchier. 2012. « Petites formes, grands desseins. D'une grammaire des énoncés éditoriaux à la standardisation des écritures ». Davallon Jean (éd.). L'économie des écritures sur le web (Paris : Hermès-Lavoisier), 165-201

Candel, Étienne \& Pergia Gkouskou-Giannakou. 2017. « Autorité et pratiques de légitimation en ligne ». Quaderni [En ligne] 93 | Printemps 2017, http://journals.openedition.org/quaderni/1066 (consulté le 25/09/2020)

Fassin, Didier. 1996. L'espace politique de la santé (Paris : PUF)

Fournout, Olivier. 2009. « La matrice relationnelle Du diatexte à l'anthropologie de la communication », Communication \& Langages 162.4, 29-48

Gagnayre, Rémi \& Olivia Gross. 2013. « Hypothèse d'un modèle théorique du patient-expert et de l'expertise du patient : processus d'élaboration », Revue Recherches Qualitatives 15 (Hors Série "les Actes »), 147-165

Georges, Fanny \& Virginie Julliard. 2018. « Produire le mort. Pratiques d'écriture et travail émotionnel des deuilleurs et des deuilleuses sur Facebook », Réseaux 210, 89-116.

Jeanneret, Yves \& Emmanuël Souchier. 1999. « Pour une poétique de l'écrit d'écran », Xoana 6, 97-107.

Jeanneret, Yves. 2014. Critique de la trivialité. Les médiations de la communication, enjeu de pouvoir (Le Havre : Non Standard)

Jolivet, Alexia. 2010. « Voir les normes comme un travail de sens : le texte comme agencement de narrations ", Études de communication 34, 75-92

Krieg-Planque, Alice. 2015. « Construire et déconstruire l'autorité en discours. Le figement discursif et sa subversion », Mots. Les langages du politique 107.1, 115-132

Lamy, Aurélia. 2017. « Mise en cause de l'autorité médicale et légitimation du discours d'expérience sur les forums de discussion en ligne ", Quaderni, DOI: 10.4000/quaderni.1074 (consulté le 25/09/2020)

Marin, Louis. 1994. De la représentation (Paris : Seuil)

Marcoccia, Michel. 2004. «L'analyse conversationnelle des forums de discussion : questionnements méthodologiques ", Les Carnets du Cediscor [En ligne], 8 | 2004, http:// journals.openedition.org/cediscor/220 (consulté le 28/09/2020)

Mayère, Anne. 2018. « Patients projetés et patients en pratique dans un dispositif de suivi à distance », Réseaux 207, 197-225

Méadel, Cécile. 2010. « Les savoirs profanes et l'intelligence du Web », Hermès 57.2, 111-117

Méadel, Cécile \& Madeleine Akrich. 2010. « Internet, tiers nébuleux de la relation patientmédecin », Les Tribunes de la santé 29, 41-48

Monte, Michèle \& Claire Oger. 2015. « La construction de l'autorité en contexte. L'effacement du dissensus dans les discours institutionnels ", Mots. Les langages du politique [En ligne], 107 | (consulté le 01/10/2020)

Mouillaud, Maurice \& Jean-François Tétu. 1989. Le Journal quotidien (Lyon : PUL) 
Oger, Claire. 2013. Discours d'autorité, discours autorisés ; faire référence et dire l'institution, Mémoire d'habilitation à diriger des recherches (Paris : CELSA Paris-Sorbonne)

Perrier, Valérie Jeanne. 2006. « Des outils d'écriture aux pouvoirs exorbitants ? Réseaux 137.3, https://www.cairn-int.info/revue-reseaux1-2006-3-page-97.htm (consulté le 26/09/2020 )

Romeyer, Hélène. 2008. " TIC et santé : entre information médicale et information de santé », tic\&société [En ligne] Vol. 2.1, https://journals.openedition.org/ticetsociete/365 (consulté le 26/09/2020)

Saemmer, Alexandra. 2016. "Sémiotique critique du discours hypertextualisé Eléments de méthodologie, à partir de l'analyse d'un livre numérique enrichi », Semen [En ligne], 42 | 2016, http://journals.openedition.org/semen/10632 (consulté le 16/10/2020)

Simon, Justine. 2016. « Présentation », Semen [En ligne], 42 | 2016, http:// journals.openedition.org/semen/10607 (consulté le 16/10/2020)

Souchier, Emmanuël, Etienne Candel, , Gustavo Gomez-Mejia \& Valérie Jeanne-Perrier. 2019. Le numérique comme écriture. Théories et méthodes d'analyse (Paris : Colin)

Spire, Antoine \& Rollon Poinsot. 2007. « L'annonce en cancérologie », Questions de communication 11, 159-176

Tourette-Turgis, Catherine. 2013. « L'effet Framingham en éducation thérapeutique : de l'enquête épidémiologique à l'enquête sur soi ", Sciences sociales et santé 31, 31-38

Wrona, Adeline \& Émeline Seignobos (éds). 2017. La Fabrique de l'autorité. Figures des décideurs en régime médiatique (Paris : Les Petits Matins)

\section{NOTES}

1. «Le patient internaute », rapport de la Haute Autorité de Santé, mai 2007, p. 21.

2. Ibid.

3. Les architextes désignent, dans les médias informatisés, des outils d'écriture qui, placés en amont du texte, conditionnent l'écriture, des objets informatiques qui régissent l'écriture, par des formats et déterminent donc la réalisation du texte (Jeanneret et Souchier 1999).

4. La notion de « diatexte » est proposée par Olivier Fournout pour qualifier « le côte-à-côte de fragments de textes " attribuables à des instances d'énonciation différentes, saisis sur une même surface matérielle » (Fournout 2009 : 29).

5. Par souci de confidentialité, nous anonymisons les médecins cités par les scripteurs. Nous faisons également le choix de la fidélité à l'expression figurant sur les forums, quelle que soit sa correction orthographique ou grammaticale.

6. https://sante-medecine.journaldesfemmes.fr/forum/affich-1655002-glioblastome

7. https://forum.doctissimo.fr/sante/cancers/glioblastome-avis-sujet_177892_1.htm

8. https://www.beaute-test.com/forums/index.php?topic=359969

9. https://sante-medecine.journaldesfemmes.fr/forum/affich-1655002-glioblastome

10. Aurélia Lamy rappelle que « l'autorité médicale semble être de fait dans une société où on considère que "l'autorité s'acquiert, au fur et à mesure où un champ de connaissances précises se dessine, et conduit à la prise de conscience que le médecin "sait" - là où les autres ne "savent" pas » (Ménard 2008 : 247). Le médecin bénéficie en ce sens d'une sorte de "domination légale rationnelle " (Weber 1971) fondée sur son statut, son expérience, ses compétences et légitimée par l'institution qu'il représente. Il apparaît en cela comme une "figure d'autorité » (Herfray 2005) face au patient (Lamy 2017). 
11. https://forum.doctissimo.fr/sante/cancers/glioblastome-avis-sujet_177892_1.htm

12. https://sante-medecine.journaldesfemmes.fr/forum/affich-1655002-glioblastome

13. https://www.allodocteurs.fr/forums-et-chats/forums/le-blog-notes-video-de-michel-etmarina/suggestion-de-sujet-sur-le-glioblastome-cancer-du-cerveau_6268.html

14. https://www.ligue-cancer.net/forum/40821_remission-ou-guerison-dun-glioblastome

15. Ibid.

16. https://www.forumcancer.ch/forum3_fr/viewtopic.php?t=1422

17. https://guerir-du-cancer.fr/temoignage-de-bernard/

18. https://forum.doctissimo.fr/sante/cancers/atteinte-glioblastome-grade-sujet_177858_1.htm

19. https://www.allodocteurs.fr/forums-et-chats/forums/le-blog-notes-video-de-michel-etmarina/suggestion-de-sujet-sur-le-glioblastome-cancer-du-cerveau_6268.html

20. https://sante-medecine.journaldesfemmes.fr/forum/affich-1655002-glioblastome

21. https://www.beaute-test.com/forums/index.php?topic=359969

22. https://sante-medecine.journaldesfemmes.fr/forum/affich-1401164-glioblastome-grade-iv

23. http://forum.doctissimo.fr/sante/cancers/glioblastome-besoin-soutien-sujet_170658_1.htm

24. https://forum.doctissimo.fr/sante/cancers/glioblastome-avis-sujet_177892_1.htm

25. Ibid.

26. https://guerir-du-cancer.fr/temoignage-de-bernard/

27. https://forum.doctissimo.fr/sante/cancers/glioblastome-avis-sujet_177892_1.htm

28. Ibid.

29. https://www.forumcancer.ch/forum3_fr/viewtopic.php?t=1422

30. https://www.beaute-test.com/forums/index.php?topic=35996

31. Les petites formes sont « des formes écrites de la médiation. [...] L'énonciateur éditorial met à disposition de l'usager des "formes" qu'il pourra manipuler et mobiliser à sa guise dans les cadres du site » (Candel, Jeanne-Perrier, Souchier $2012: 167$ ).

32. Dans son glossaire, Yves Jeanneret définit le « signe passeur » comme suit : « Type de signe propre aux écrits d'écran qui repose sur un triple processus de production du sens - intégrer un signe particulier au texte présent à l'écran, le marquer comme susceptible d'être activé, anticiper à travers lui le texte destiné à être affiché » $(2014: 15)$.

33. https://guerir-du-cancer.fr/temoignage-de-frederic-glioblastome-grade-iv/

34. Ibid.

35. https://forum.doctissimo.fr/sante/cancers/atteinte-glioblastome-grade-sujet_177858_1.htm

36. https://forum.doctissimo.fr/sante/cancers/atteinte-glioblastome-grade-sujet_177858_1.htm

37. https://www.allodocteurs.fr/forums-et-chats/forums/le-blog-notes-video-de-michel-etmarina/suggestion-de-sujet-sur-le-glioblastome-cancer-du-cerveau_6268.html

\section{ABSTRACTS}

This paper aims to question the " parent expert " notion, through an analysis of web discourses which focus on a very specific type of cancer: glioblastoma. It is a rare brain cancer more than often diagnosed at a late stage, with a very poor prognosis and huge and disabling neurological impairments at the time of identification of the disease. Thereby, when a web patient chat room happens to deal with glioblastoma, the discussion happens most of the time between close relatives. Therefore, this paper deals with the following questions: how do these discourses show 
the parents' building of authority? To what extent is their on-line quest for authority also a try to free themselves from the medical «already-said», by requesting a kind of "never-said»? Through the semio-communicational analysis of a specific corpus of web forums, the paper underlines how parents position themselves relative to other actors and how they produce their own « authorization process ».

Cet article propose d'interroger la notion de «parent expert» autour de l'analyse de discours numériques portant sur un cancer bien particulier : le glioblastome. Il s'agit d'un cancer rare et peu connu qui, au moment du diagnostic, est souvent très avancé avec un pronostic sombre et des atteintes neurologiques déjà handicapantes. De ce fait, il s'avère que ce sont avant tout des proches qui s'expriment à son sujet au sein des «forums de patients ». Ainsi, cette contribution propose-t-elle de traiter les questions suivantes: comment les discours numériques relatifs au glioblastome font-ils apparaître la construction, par ces parents proches, de figures et positions d'autorité ? Dans quelle mesure leur quête d'autorité en ligne s'articule-t-elle à une prétention à s'affranchir du « déjà-dit » de l'institution médicale, notamment, en revendiquant $d u$ "jamais dit " ? À partir de l'analyse sémio-discursive des récits et discours tissés dans l'espace numérique d'un corpus de forums francophones dédiés, l'article montre comment les participants se positionnent par rapport aux acteurs évoqués et en viennent à produire eux-mêmes des formulations d'autorité qui s'articulent à l'expertise.

\section{INDEX}

Mots-clés: autorité, cancer, forum, parent, témoignage

Keywords: authority, cancer, forum, parent, testimony

\section{AUTHORS}

\section{JULIETTE CHARBONNEAUX}

GRIPIC, Celsa Sorbonne-Université

\section{KARINE BERTHELOT-GUIET}

GRIPIC, Celsa Sorbonne-Université 